\title{
POTENCIAL USO DE NANOMATERIALES COMBINADOS CON ÁCIDO POLILÁCTICO (PLA) EN PRÓTESIS ORTOPÉDICAS: UNA REVISIÓN
}

\author{
POTENTIAL USE OF NANOMATERIALS COMBINED WITH \\ POLYLACTIC ACID (PLA) IN ORTHOPEDIC PROSTHESES:
}

\section{A REVIEW}

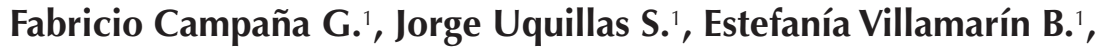 Gabriela Yánez-Jácome², David Romero-Estévez² \& Fernanda Pilaquinga F. ${ }^{1,3 *}$}

Recibido: 16 de noviembre 2020 / Aceptado: 22 de diciembre 2021 DOI 10.26807/ia.v10i1.204

Palabras clave: ácido poliláctico, biocompatibilidad, nanomateriales, PLA, prótesis.

Keywords: biocompatibility, nanomaterials, PLA, polylactic acid, prosthetics.

1 Universidad Internacional SEK, Nanotecnologías y Materiales Funcionales para Diseño, Maestría en Diseño Industrial y Procesos, Quito, Ecuador (fgcampana.mdin@uisek.edu.ec; jpuquillas.mdin@uisek .edu.ec; jevillamarin.mdin@uisek.edu.ec; *correspondencia: maria.pilaquinga@uisek.edu.ec)

2 Pontificia Universidad Católica del Ecuador, Centro de Estudios Aplicados en Química, Quito, Ecuador (gsyanez@puce.edu.ec; dfromero@puce.edu.ec)

3 Pontificia Universidad Católica del Ecuador, Facultad de Ciencias Exactas y Naturales, Escuela de Ciencias Químicas, Quito, Ecuador (mfpilaquingaf@puce.edu.ec) 


\section{RESUMEN}

Los materiales tradicionales derivados del petróleo como el polietileno y polipropileno son los más empleados en el campo de la protésica, sin embargo, la tendencia a la protección del medio ambiente amerita el análisis de nuevos materiales biodegradables y biocompatibles. El ácido poliláctico (PLA) es un polímero obtenido a partir de fuentes naturales, que se usa entre muchas aplicaciones en prótesis ortopédicas por su bajo costo. Con el desarrollo de la nanotecnología, pueden incorporarse nanopartículas de diferente naturaleza con el objetivo de mejorar las propiedades mecánicas del PLA, sin embargo, existe información limitada. En este compendio se describen las propiedades físicas y químicas del PLA, así como las condiciones para su impresión en 3D. Se analizan varios nanomateriales tales como nanocelulosa, nanofibras de quitosano, nanotubos de carbono, grafeno, óxido de titanio, nanopartículas de óxido de hierro, nanopartículas de plata, nanopartículas de sílice mesoporosa, entre otras. Se describen estudios referentes sus propiedades químicas, mecánicas y biocompatibilidad. Como resultados se encontró que algunos nanomateriales han sido combinados con PLA, los estudios realizados para comparar sus propiedades mecánicas, muestran mejores resultados usando nanopartículas. Muy pocos estudios in vivo se han realizado, únicamente nanotubos de carbono, grafeno, óxido de titanio, nanopartículas de plata y nanopartículas de sílice mesoporosa. Se concluye que el uso de nanomateriales puede mejorar potencialmente las propiedades mecánicas del PLA, sin embargo, se requieren los estudios experimentales correspondientes, además que se deben usar modelos animales para evaluar su efecto a nivel tisular y determinar si son aptos para la combinación o recubrimiento de prótesis.

\section{ABSTRACT}

Traditional petroleum-derived materials like polyethylene and polypropylene are the most commonly utilized in the field of prosthetics, but the movement toward environmental conservation necessitates the investigation of novel biocompatible and biodegradable materials. Due to its low cost, polylactic acid 
(PLA) is a polymer derived from natural sources that is utilized in a variety of applications, including orthopedic prosthesis. Nanoparticles of various natures can be added to improve the mechanical characteristics of PLA with the advancement of nanotechnology, although there is insufficient information. The physical and chemical characteristics of PLA, as well as the requirements for 3D printing with it, are described in this review. Nanocellulose, chitosan nanofibers, carbon nanotubes, graphene, titanium oxide, iron oxide nanoparticles, silver nanoparticles, and mesoporous silica nanoparticles are among the nanomaterials studied. The chemical, mechanical, and biocompatibility aspects of the material have been studied. As a result, some nanomaterials have been mixed with PLA, and studies comparing their mechanical characteristics reveal that nanoparticles produce better outcomes. Very few in vivo studies have been performed, only carbon nanotubes, graphene, titanium oxide, silver nanoparticles and mesoporous silica nanoparticles. The introduction of nanoparticles could possibly enhance the mechanical properties of PLA, but further research is needed, and animal models should be utilized to evaluate their effect at the tissue level and decide whether they are acceptable for combination or coating prostheses.

\section{INTRODUCCIÓN}

Una prótesis es un dispositivo que sustituye a un miembro del cuerpo y realiza casi las mismas funciones originales. Las prótesis son elaboradas principalmente de polietileno $(\mathrm{PE})$, polipropileno (PP) y poliuretano. Los reemplazos de cadera y rodilla incluyen porciones hechas de metal como acero inoxidable, tita- nio o cromo y aleaciones de cobalto (ArthritisFoundation, 2016). Actualmente, por la tendencia a la reducción de la contaminación ambiental, el uso prótesis a base de materiales obtenidos a partir de recursos renovables se ha convertido en una realidad. 


\section{Ácido poliláctico (PLA)}

El ácido poliláctico o poliácido láctico (PLA) es un polímero clasificado como un poliéster alifático (Liao et al., 2019). Su unidad básica es el ácido láctico o ácido 2-hidroxipropanoico (Figura 1).

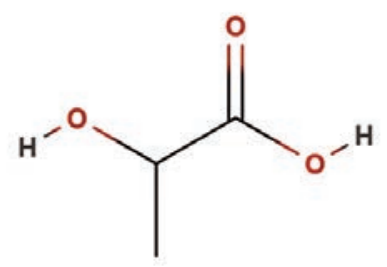

Figura 1. Estructura química del PLA

Químicamente es un material inerte y se obtiene a partir de fibras naturales como maíz, trigo y arroz, por lo que es amigable con el ambiente, biodegradable, reciclable y compostable. La lactida, un monómero principal del PLA, presenta quiralidad (L-lactida o D-lactida), lo que determina su biodegradabilidad y propiedades mecánicas finales, así como, su estructura semicristalina o amorfa, respectivamente. En general, las formas $\mathrm{D}$ y $\mathrm{L} / \mathrm{D}$ se degradan más rápidamente que la forma $L$. Su metabolismo permite que el organismo lo asimile por su conversión a dióxido de carbono (Sha et al., 2016). El PLA es utilizado en películas plásticas, botellas, envases para alimentos (Gregor et al., 2017; Nagarajan et al., 2016) y en aplicaciones médicas como en exoprótesis, hilos de sutura, por su alta resistencia, degradabilidad y biocompatibilidad (Casalini et al., 2019; Ghasemi et al., 2018). El tiempo estimado de degradación es de 6 a 12 meses (Kucharczyk et al., 2016) y su vida media en estado puro es de 30 semanas, sin embargo, esto puede modificarse en función de las necesidades clínicas, ajustando la composición molecular y la arquitectura física de la prótesis (da Silva et al., 2018).

El PLA es un polímero hidrolizable en agua, además, presenta actividad antibacterial (Liao et al., 2019; Parwe et al., 2014). Su semipermiabilidad al agua y al oxígeno lo hacen más suceptible a la degradación (da Silva et al., 2018). La difusión de agua en la estructura interpolimérica del PLA, degrada su microestructura interna, debido a la formación de cavidades (Göpferich, 1996). Su degradación también depende en mayor proporción del pH y la tem- 
peratura (L. Xu et al., 2011). Otros de los mecanismos que contribuyen a su degradación, son los procesos enzimáticos asociados con una infección o procesos de inflamación (da Silva et al., 2018).

La relación de la cantidad de PLA en el material, la presencia de otros materiales y su arquitectura molecular, están directamente relacionadas con sus compatibilidad química y propiedades mecánicas (Oksiuta et al., 2020). En la Tabla 1 se muestran las propiedades mecánicas del PLA.

Tabla 1. Propiedades mecánicas del PLA (Vargas Pérez et al., 2015)

\begin{tabular}{cc}
\hline Propiedad & Unidad \\
\hline Límite elástico & $49 \mathrm{MPa}$ \\
\hline Elongación & $2,5 \%$ \\
\hline Módulo Elástico & $3,2 \mathrm{GPa}$ \\
\hline Resistencia a la flexión & $70 \mathrm{MPa}$ \\
\hline Módulo de tensión & $3-4 \mathrm{GPa}$ \\
\hline Fuerza de tensión & $40-60 \mathrm{MPa}$ \\
\hline
\end{tabular}

El PLA se contrae con el calor, por lo que es adecuado como material de envoltura retráctil. Su punto de fusión es $154{ }^{\circ} \mathrm{C}$ (Hou \& Qu, 2019). Por su baja temperatura de transición vítrea, no es adecuado para retener líquidos calientes. Su manufactura no requiere sistemas complejos, generalmente se usa mediante moldeo por inyección, soplado, extrusión, termoformado, hilado de fibras o formación de película (Auras et al., 2004). Por su procesabilidad requiere un cuarto de energía, comparado con los polímeros obtenidos a base de petróleo como el PE o PP (Casalini et al., 2019).

\section{Moldeo por inyección en 3D}

Esta tecnología permite generar formas geométricas personalizadas. El proceso es relativamente sencillo, se requiere una impresora de $3 \mathrm{D}$ o de corte láser y el filamento de PLA. Se parte de un diseño en 3D, existe una variedad de programas para diseñar prótesis, sin embargo, el software Tinkercad (https://www.tinkercad.com) permite usar piezas gratuitas previamente diseñadas. El archivo del diseño se guarda en un pendrive en formato .obj, .stl, .glb o .svg y se conecta a la impresora 3D. Los parámetros de impresión se muestran en la Tabla 2. 
Tabla 2. Parámetros de impresión 3D usando un filamento de PLA (Liv et al., 2017)

\begin{tabular}{lc}
\hline Parámetro & Valor \\
\hline Temperatura del extrusor $\mathrm{T}_{\mathrm{e}}\left({ }^{\circ} \mathrm{C}\right)$ & 220 \\
$\begin{array}{l}\text { Temperatura de la placa } \\
\text { e impresión } \mathrm{T}_{\mathrm{b}}\left({ }^{\circ} \mathrm{C}\right)\end{array}$ & 60 \\
Velocidad durante extrusión $\mathrm{V}_{\mathrm{e}}(\mathrm{mm} / \mathrm{s})$ & 60 \\
Velocidad sin extrusión $\mathrm{V}_{\dagger}(\mathrm{mm} / \mathrm{s})$ & 120 \\
$\begin{array}{ll}\text { Distancia entre la punta de la boquilla } \\
\text { y la capa de construcción d }(\mathrm{mm})\end{array}$ & 0,1 \\
Proporción de llenado $\mathrm{F}(\%)$ & 100 \\
\hline
\end{tabular}

El proceso inicia con la introducción del PLA mediante una boquilla que se va calentando gradualmente, el material semi-fundido se irá depositando sobre la mesa de trabajo con una temperatura menor capa por capa de manera controlada (Ñauta Ñauta \& Vergara Idrovo, 2017). Con esta técnica, no se generan desechos asociados a la producción, se considera sostenible y es un 90 \% más rápido en la obtención de piezas, por lo que esta tecnología ha experimentado un crecimiento exponencial en los últimos años (Gómez Blázquez, 2019).

\section{Ensayos de propiedades mecánicas de PLA}

Dentro de las principales características mecánicas asociadas a la evaluación del grado de inalterabilidad del PLA (o mezcla), para establecer una durabilidad y biocompatibilidad del producto final, están la determinación de pérdida de masa, grado de cristalinidad, energía termogravimétrica y de activación, dureza y tracción, resistencia a fractura, y tensión para deformación, entre otras (Oksiuta et al., 2020; Qiu et al., 2016).

Determinación de pérdida de masa La pérdida de masa de los materiales se determina mediante una comparación entre la diferencia del peso inicial y el peso final seco. Las muestras se colocan dentro de una incubadora a una temperatura de $37,0 \pm$ $0,5{ }^{\circ} \mathrm{C}$, simulando la temperatura corporal, durante 180 días (Oksiuta et al., 2020).

\section{Grado de cristalinidad}

La temperatura de cristalización, grado de cristalinidad, temperatura de fundición, entalpía de cristalización en frío y entalpía de fusión de 
los materiales utilizados en prótesis, se identifican mediante la calorimetría diferencial de barrido (CDB). El grado de cristalinidad se calcula mediante fórmulas matemáticas con los parámetros determinados en las curvas de CDB (Qiu et al., 2016).

\section{Energía termogravimétrica y de ac- tivación}

La termogravimetría se realiza mediante analizadores con incrementos de temperatura, donde los materiales son sometidos a descomposición térmica (Oksiuta et al., 2020). Por otra parte, la energía de activación se determina mediante el método Kissinger, en donde se estudia la calorimetría diferencial de barrido, análisis térmico diferencial y la termogravimetría derivativa (Vyazovkin, 2020). Este método se basa en la dependencia de la temperatura sobre el rango de calentamiento de los materiales.

\section{Dureza y tracción}

Los ensayos de dureza se realizan en base a la norma estándar ASTM D2240 (Cakir et al., 2018). El empleo de esta norma permite evaluar la dureza de varios tipos de durómetros (A, D y M). Para el caso del PLA, se emplea el durómetro tipo D (Cakir et al., 2018; Oksiuta et al., 2020).

El comportamiento mecánico de los materiales se determina mediante ensayos de tracción, a través del módulo de Young, límite elástico y alargamiento a la rotura (Farah et al., 2016). Estas propiedades específicas son importantes ya que determinan las dimensiones necesarias para una cierta resistencia mecánica o rigidez. Así mismo, la resistencia a la tracción, el módulo elástico y el alargamiento porcentual pueden ser determinados mediante la norma estándar ASTM D638 (Anand Kumar \& Shivraj Narayan, 2019; Cakir et al., 2018).

\section{Uso de nanomateriales con PLA como matriz polimérica}

El PLA presenta baja resistencia mecánica, hidrolizabilidad y mala adherencia celular, por lo que podría causar no sólo fractura de la prótesis, sino infecciones en el organismo. Para su uso en exoprótesis se emplea un recubrimiento de silicona médica (Komoto et al., 2018). La nanotecnología permite manipular estructuras 
a nanoescala, creando materiales más resistentes utilizados en prótesis, implantes, ingeniería de tejidos, componentes de órganos artificiales, entre otros (Mediforum, 2016). Los estudios sobre el uso materiales para mejorar las propiedades mecánicas del PLA son limitados (Farah et al., 2016). En el presente estudio se analizan varios nanomateriales que se han empleado para mejorar las propiedades mecánicas de PLA y que pueden ser potencialmente usadas in vivo. Estudios de adaptación de la superficie de materiales han permitido desarrollar metodologías para anclar nanomateriales solos o en combinación con otros sobre superficies de prótesis, incorporando funcionalidades específicas adicionales que permiten superar las carencias del material en cuanto a la interacción con el medio, pero manteniendo las propiedades del material de partida (Petersen et al., 2014). La incorporación de los nanomateriales en estado sólido (polvo) sobre PLA, puede realizarse en disolución en cloroformo, éter de petróleo o etanol, se elimina el disolvente y se coloca sobre el molde del filamento para la impresión en 3D (aprox. 1,75 $\mathrm{mm})$. La mezcla también puede re- alizarse por extrusión o inyección, con maquinaria usada para la elaboración de plásticos (Ziabką et al., 2018). A continuación de describen los nanomateriales usados en combinación con el PLA:

\section{Nanocelulosa}

La celulosa es un polímero estructural presente en la naturaleza, forma parte de las plantas confiriendo sus propiedades mecánicas (Dufresne, 2013). Es considerado el polímero renovable más abundante. La estructura de la celulosa está compuesta por unidades de $\beta$-1,4-anhydro-Dglucopiranosa. Los grupos hidroxilos tienen la habilidad de formar puentes de hidrógeno y esta característica otorga que la estructura sea fibrilar y semicristalina, lo que influye directamente en sus propiedades físicas y mecánicas (French, 2014). En la actualidad se han desarrollado nanomateriales a partir de la celulosa. Existen tres tipos de nanocelulosa, nanocristales de celulosa (CNC), nanofibrillas de celulosa (CNF) y celulosa bacteriana (BC) (Lin \& Dufresne, 2014). A nivel industrial, existen varios proyectos con el objetivo de producir grandes cantidades de celulosa microfibrilada con caracterís- 
ticas uniformes. En un estudio realizado por Tanpichai (Tanpichai \& Wootthikanokkhan, 2016) usando una mezcla de fibras de bamboo y PLA, tanto el módulo de Young como la resistencia a la tensión mejoraron en un $42 \%$ y $18 \%$, de 32,8 MPa a 38,7 MPa y de 2,4 a 3,4 GPa respectivamente.

Se ha reportado que materiales celulósicos pueden causar respuestas leves in vivo (Gumrah Dumanli, 2017), pues la celulosa no es degradada por el cuerpo humano ya que no posee enzimas celulósicas. Existen pocas investigaciones enfocadas a estudiar la biocompatibilidad de la nanocelulosa (Lin \& Dufresne, 2014).

A nivel de nanopartículas existe un gran desafío para encontrar una dispersión homogénea en su matriz polimérica, debido a que los grupos hidroxilos de la superficie interactúan causando agregación, mientras menor es el tamaño de la partícula este fenómeno se magnifica, limitando el potencial del refuerzo mecánico (Dufresne, 2013). Pocas investigaciones se han encontrado acerca del uso de la nanocelulosa y
PLA en prótesis, Zhou et al. (2013) fabricaron andamios de bio-nanocompósitos fibrosos electrohilados con CNCs utilizando anhídrido maleico injertado en PLA como matriz. Su biocompatibilidad fue analizada utilizando células madres mesenquimales derivadas de tejido adiposo humano. El nanomaterial demostró una estabilidad mejorada durante la degradación in vitro, no resultó ser tóxico para las células, las cuales fueron capaces de soportar la proliferación celular. Los autores concluyeron que este nanomaterial posee propiedades útiles para la ingeniería de tejidos óseos. Actualmente, se investiga el uso de materiales a base de nanoquitina-nanocelulosa, este material posee un alto rendimiento debido a su gran área superficial y a su tamaño pequeño (Kalarikkal et al., 2016). Se usó la línea celular de fibroblastos de un roedor para investigar su biocompatibilidad, mostró ser más susceptible a la bioadhesión a este nanomaterial y por tanto, a su proliferación en comparación a otros nanomateriales (Ee \& Yau Li, 2021).

\section{Quitosano}

El quitosano es el polímero natural más abundante después de la celu- 
losa (Roberts, 1992). La quitina se encuentra presente en los exoesqueletos de los crustáceos. Mediante la $\mathrm{N}$-desacetilización, la quitina se puede transformar en quitosano (Hernández-Cocoletzi et al., 2009). La principal fuente de obtención es la industria camaronera, el aprovechamiento de este material está ayudando a disminuir millones de toneladas de desperdicios a nivel mundial. Químicamente, es un polímero policatiónico compuesto por unidades de $\beta$-1,4-D-glucosamina (El-Ghaouth et al., 2000). Las propiedades fisicoquímicas del quitosano como la biodegradabilidad, biocompatibilidad, ausencia de toxicidad y naturaleza antimicrobiana, han permitido que se lo emplee en distintas áreas como medicina, cosmética, farmacia, agricultura, alimentación, entre otras (Hernández-Cocoletzi et al., 2009). Las nanopartículas de quitosano son materiales coloidales con un tamaño entre 1 y 1000 nm, presentan alta estabilidad y reactividad, adsorción y facilidad de alcanzar los tejidos diana (Carmona et al., 2018). Durante las últimas décadas, se ha investigado sobre la biocompatibilidad del quitosano, demostrando que éste no es tóxico, y puede soportar una variedad de células para que se adhieran y proliferen (Sangeetha et al., 2019). Entre éstas se encuentran los osteoblastos, condrocitos, fibroblastos, células del núcleo pulposo, células neurales y endoteliales; además, ha sido comprobado su potencial para ser utilizado en hueso, cartílago, piel, disco intervertebral, ligamento y tendón, y en la ingeniería de tejidos nerviosos y vasculares (Jiang et al., 2014). Usando nanopartículas de quitosano con PLA como matriz, se observó un aumento de elasticidad medida a través del módulo de Young (Sonseca et al., 2019). Así mismo, los copolímeros a base de PLA/quitosano mostraron una mayor resistencia del material y un tiempo de degradación prolongado. Los estudios in vivo indicaron una resistencia suficiente del PLA durante 8-12 semanas después de la implantación. Estos hallazgos sugieren que el PLA puede usarse eficazmente para la fijación interna de huesos (DeStefano et al., 2020). La mayor parte de mezcla PLA/quitosano se emplea como transportadores de fármacos (Dev et al., 2010). 


\section{Nanotubos de carbono}

Son láminas de capas de grafito enrolladas de manera concéntrica. Existen dos tipos de nanotubos de carbono (CNT por sus siglas en inglés), dependiendo de las capas de grafito que forman, nanotubos de carbono de pared múltiple (MWCNT por sus siglas en inglés) y nanotubos de carbono de pared sencilla (SWCNT por sus siglas en inglés) (Figura 2) (Andrade Guel et al., 2012). Fueron descubiertos por primera vez en 1991 y los primeros nanocompuestos poliméricos usando nanotubos de carbono se reportaron en 1994, los cuales poseen baja densidad, alta flexibilidad, resistencia, conductividad térmica (Moniruzzaman \& Winey, 2006).

Los CNTs pueden Ilegar a ser más fuertes que el acero, más ligeros que el aluminio y con mayor conductividad que el cobre (Enyashin \& Ivanovskii, 2007; Moniruzzaman \& Winey, 2006).

Los híbridos formados entre biomoléculas (ADN, proteínas) con CNTs son utilizados en el campo biomédico (Dresselhaus et al., 2000).
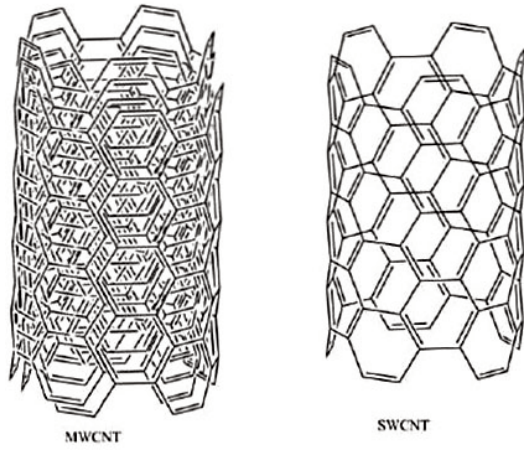

Figura 2. Representación nanotubos de Guel et al., 2012)

La mayor parte de investigaciones acerca del uso de nanotubos de carbono en el área de la protésica, emplean hidroxiapatita (HA) como material de recubrimiento. En un estudio in vivo de CNTs y recubrimiento de $\mathrm{HA}$ en implantes de titanio incrustados en huesos de roedores, no se observó ningún efecto fisiológico adverso o citotoxicidad en tejidos y células óseas (Facca et al., 2011). La adición de CNTs indujo una mayor osteointegración como en comparación con HA. El módulo de elasticidad del hueso nuevo se comparó con el módulo de HA-CNTs/hueso para comprender la integridad mecánica del implante, siendo el modificado con CNTs más elástico. Pocos estudios analizan su 
combinación con PLA, pero no se reportan estudios in vivo. Flores-Cedillo y colaboradores usaron una matriz de PLA con CNTs de pared múltiple para simular un andamio para la regeneración ósea. No se realizaron ensayos biológicos, únicamente mecánicos, que mostraron una diferencia muy significativa en módulo de Young de $6000 \mathrm{MPa}$ usando nanotubos y PLA (Ángellópez, 2017).

Los CNTs pueden promover un microambiente similar a la matriz extracelular, por lo que lo hace un potencial candidato para el desarrollo de andamios artificiales (Kalarikkal et al., 2016). La biocompatibilidad de los CNTs mejora cuando se los incorpora a una matriz de PLA, esto puede prometer un buen futuro en los sistemas biomédicos y en el desarrollo de bio-nanomateriales, además, se asegura que la preparación de estos nanocompuestos es apta para la industria pues son mucho más baratos y requieren de menos pasos para su fabricación (Akbari et al., 2015).

\section{Grafeno}

Es una lámina fina plana de carbono con una estructura de retícula hexagonal en 2D con hibridación $\mathrm{sp}^{2}$. Entre sus propiedades destaca un valor de módulo de Young elevado, gran superficie teórica, buena transparencia óptica y una excelente conductividad térmica (Huang et al., 2012). En la actualidad, se ha logrado combinar compuestos a base de grafeno con polímeros, biomateriales, nanoestructuras inorgánicas, cristales orgánicos y CNTs (Huang et al., 2012). Una base polimérica de PLA junto con grafeno aumentó hasta en un $160 \%$ en relación a la resistencia a la tracción (Kucharczyk et al., 2016).

El grafeno ha mostrado un potencial excepcional para usos biológicos, uno de éstos es la regeneración ósea. Diferentes tipos de células de osteoblastos han sido testeados con el grafeno para este tipo de aplicaciones. Además, se ha comprobado su biocompatibilidad, usando capas de grafeno y lamininas, puede promover la adhesión celular y la configuración celular pseudopodial (Kalarikkal et al., 2016). La biocompatibilidad puede verse afectada por los materiales de partida que se usen y los métodos que se apliquen para la 
producción de materiales a base de grafeno. Cuando estos materiales son incorporados al PLA por ejemplo, las propiedades mecánicas del polímero mejoran, siendo importante en el área de la protésica e implantes quirúrgicos. Cultivos de fibroblastos de embriones de roedor fueron testeados para evaluar la biocompatibilidad de películas compuestas por dos materiales de grafeno: óxido de grafeno y nanoplaquetas de grafeno incorporadas en PLA. Las películas de PLA/óxido de grafeno mostraron una mayor adhesión y proliferación celular. El óxido de grafeno en la superficie del material incrementó su hidrofilicidad, formando una morfología de superficie adecuada para la adsorción proteica y adhesión celular (Pinto et al., 2013).

\section{Óxido de titanio}

El óxido de titanio $\left(\mathrm{TiO}_{2}\right)$ se presenta naturalmente como tres minerales conocidos como rutilo, anatasa y brookita. Es uno de los materiales más blancos que existen, es por esto que se lo ha utilizado en cosméticos como protector solar, además se lo incorpora en pinturas en materiales de construcción, fibras de tela y papel (Kasuga et al., 1998; Peters et al., 2014). La dispersión de la luz alcanza su máxima eficiencia en partículas de 200 a 300 nm de diámetro (Yan et al., 2010). Las películas delgadas de $\mathrm{TiO}_{2}$ con estructuras nanoporosas tienen gran superficie y alta reactividad (Gong et al., 2001). Las $\mathrm{TiO}_{2}$ NPs no son tóxicas, por esta razón tienen grandes usos en recubrimientos antibacterianos (Amarnath et al., 2013). Varios estudios indican que un contenido mínimo de $\mathrm{TiO}_{2}$ en una matriz de PLA, incrementan la resistencia del material en relación a la fractura (Segura González, 2016). $\mathrm{El} \mathrm{TiO}_{2}$ tiene notables propiedades físicas y químicas como la estabilidad, sensibilidad y selectividad, y biológicas como la biocompatibilidad, las cuales lo han convertido en un material adecuado para distintas aplicaciones biológicas (Kumar et al., 2018). Shebi \& Lisa (2019) a partir del extracto de la planta Piper nigrum fabricaron $\mathrm{TiO}_{2}$ NPs mediante el método de síntesis sol-gel, para añadirlas a PLA. Su biocompatibilidad fue evaluada usando las células de fibroblasto de roedor, demostrando que existe una elevada viabilidad celular debido a la presencia de partículas de $\mathrm{TiO}_{2}$ en las películas de PLA. Se concluyó que, debido al 
tamaño de los poros, se puede variar significativamente los comportamientos celulares como la adhesión, propagación o proliferación.

Dentro de la cirugía ortopédica y dental, los implantes a base de Ti son la primera opción por su alta biocompatibilidad. Sin embargo, se requiere modificar la superficie del implante, para obtener una fuerte osteointegración con el hueso. Las nanopartículas de $\mathrm{TiO}_{2}$ son un material de recubrimiento prometedor para la mejora de la actividad osteogénica alrededor de los implantes (Ahn et al., 2019).

Rajapakse y su grupo describieron una técnica novedosa, simple y de bajo costo para preparar metal de titanio recubierto de hidroxiapatita, a través del crecimiento de capas delgadas de nanopartículas de $\mathrm{TiO}_{2}$ autoformadas sobre superficies metálicas de titanio. Este estudio demostró ser altamente útil para preparar implantes ortopédicos de bajo costo a gran escala., sin riesgo citotóxico (Rajapakse et al., 2016) .

Nanopartículas de óxido de hierro Las nanopartículas de óxido de hierro $\left(\mathrm{Fe}_{3} \mathrm{O}_{4}\right)$ han captado mucha aten- ción debido a sus propiedades superparamagnéticas, como la orientación magnética de fármacos, la resonancia magnética y los catalizadores (Zaitsev et al., 1999). La aplicación del suministro de fármacos es de particular interés para estas partículas por su biocompatibilidad. El uso de un campo magnético externo podría permitir el transporte de fármacos directamente a tejidos específicos (Chomoucka et al., 2010). Las nanopartículas de $\mathrm{Fe}_{3} \mathrm{O}_{4}$ tienen la capacidad de mejorar el contraste de resonancia magnética y mejorar los sistemas de administración de fármacos. También se ha informado que las nanopartículas magnéticas distribuidas en una matriz de polímero biodegradable, se liberan de manera controlada en relación al tiempo y ubicación (Wassel et al., 2007). Pocos estudios sobre el uso de nanopartículas de hierro con PLA en prótesis ha sido estudiada, su objetivo se basa en generar memoria reprogramable inducida magnéticamente en un derivado de PLA, con ácido poliD, L-láctico (PDLLA), pudiendo aplicarse a prótesis no deformables (X. Zheng et al., 2009), o como agentes transportadores de fármacos usando PLA (Xiang et al., 2017). En la inves- 
tigación de Zhou et al. (2013) se fabricaron y caracterizaron andamios de cristales de nanocelulosa (CNC) y anhídrido maleico (MAH) injertados en PLA, como matriz (MPLA/ CNC). Esta investigación demostró por primera vez que la interacción de estos bio-compuestos puede promover la formación de microestructuras nanofibrosas de matriz extra- celular, así como una mejora de las propiedades mecánicas y citocompatibilidad.

\section{Nanopartículas de plata}

Las nanopartículas de plata (AgNPs) poseen propiedades antibacterianas y baja toxicidad (Chen \& Schluesener, 2008). Se han reportado estudios realizados en AgNPs utilizando diferentes polímeros; éstos incluyen la síntesis en polivinilpirrolidona (Zheng et al., 2001), alcohol polivinílico (Khanna et al., 2005), poliuretano hiperramificado (Lu et al., 2003) y poliacrilonitrilo (Zhang, 2001). Existe limitada información sobre el uso de AgNPs y PLA en prótesis y sus correspondientes ensayos in vivo. En un estudio de propiedades mecánicas entre AgNPs/quitosano y PLA, se obtuvo un rendimiento superior al usar nanopartículas en relación al Módulo de Young con un valor de 88 a $256 \mathrm{MPa}$. (Sonseca et al., 2019). Acerca de los ensayos realizados in vivo, usando una pequeña copa como implante en el músculo de un roedor, se ha analizado el efecto de AgNPs con otro tipo de polímero, acrilonitrilo butadieno estireno (ABS) (Ziabką et al., 2018). Los estudios in vivo confirmaron la biocompatibilidad de los implantes, tanto el ABS puro como el modificado con nanopartículas, exhibieron una clara disminución en el área de granulación, misma que fue reemplazado por tejido muscular en regeneración. Se observó un área ligeramente más pequeña de tejido de granulación en los alrededores de la prótesis con AgNPs que con ABS puro. Sobre las propiedades mecánicas, la fuerza de tensión fue ligeramente mayor (54 MPa), en comparación a la prótesis a $A B S$ puro con $52 \mathrm{MPa}$. En la investigación de Gherasim et al. (2020), fabricaron compósitos nanoestructurados recubiertos de PLA/AgNPs mediante la técnica de vaporación láser pulsada asistida por matriz. Se demostró que este material resultó ser biocompatible in vitro con células endoteliales humanas y que puede ser usado para aplicaciones biomédicas. 
Nanopartículas de sílice mesoporosa Las nanopartículas de sílice mesoporosa (MSN) son una de las nanopartículas inorgánicas mejor estudiadas para la administración de fármacos y agentes de contraste ( $\mathrm{Xu}$ et al., 2019) Estas nanopartículas poseen algunas características únicas, como una alta capacidad de carga de fármaco debido a su gran área de superficie y alto volumen de poros, así como al tamaño de partículas y poros sintonizables. Para administrar agentes de ácido nucleico, la superficie de sílice se convierte para transportar cargas positivas. Las nanopartículas de sílice mesoporosa han sido empleadas con éxito como recubrimiento de prótesis e implantes dentales (Sangeetha et al., 2019). Sin embargo, en combinación con PLA, evidenció que la adición de nanopartículas genera una alteración en su degradación térmica, debido a la dependencia del tiempo, temperatura y estructura del polímero. La utilización de estas nanopartículas mejoró sus propiedades mecánicas presentando, una dependencia directa del contenido de sílice (Botia Prada \& Orjuela Abril, 2016).
Referente a la biocompatibilidad, las NPs de sílice pueden ocasionar lesiones intracelulares causadas por la interacción entre éstas y los sistemas biológicos, los cuales incluyen peroxidación de la membrana, agotamiento del glutatión, disfunción mitocondrial y/o daño del ADN. Sin embargo, todos estos sucesos están relacionados al tipo de la célula, a las características de las NPs o en la forma como ellas interactúan. Las características son tamaños a nanoescala, estructuras mesoporosas, área superficial, volúmenes largos del poro. Las nanopartículas de sílice no han mostrado citotoxicidad en muchos sistemas biológicos con las adecuadas características y correctas dosis. Existe un amplio rango por investigar sobre la biocompatibilidad de estas nanopartículas (Asefa \& Tao, 2012).

\section{Otros tipos de nanomateriales}

Las nanopartículas de sepiolitas en una matriz de PLA, lograron disminuir los efectos de degradación del polímero al reducir la movilidad de sus cadenas por miscibilidad (Velasco Nieto, 2016). 
Por otro lado, las nanopartículas de colágeno pueden ser utilizadas en combinación con componentes matriz como PLA, con el objetivo de mejorar la proliferación y el crecimiento vascular proporcionando un sustrato para la regeneración epidérmica. Las mallas poliméricas sintéticas demuestran buena biocompatibilidad, altas propiedades mecánicas y son de fácil manejo. Sin embargo, los andamios de polímeros sintéticos tienen problemas de hidrofobicidad superficial y pérdida de células sembradas (Colorado et al., 2013).

La biocompatibilidad y la biodegradabilidad del colágeno han aportado sin duda un gran potencial hacia el campo de la ingeniería de tejidos. Geles nanofibrilares de colágeno fueron preparados para apoyar la unión de células neuronales y para la reparación del cerebro; sustitutos de la piel basados en el cultivo de células sobre andamios 3D de colágeno actualmente, son comercializados. El colágeno ha resultado ser biocompatible para la mayoría de tejidos como huesos, tendones, vasos sanguíneos, piel, córnea, entre otros. Además, éste estimula el crecimiento celular y modifica la morfología, migración, adhesión y diferenciación celular (Kalarikkal et al., 2016).

\section{CONCLUSIÓN}

Aunque el uso de PLA en el área de la protésica es común, por su baja resistencia mecánica, es necesario el estudio para poder mejorarla. En la revisión bibliográfica realizada se citaron varios tipos de nanomateriales, de diferente naturaleza, algunos de ellos se han ensayado con PLA a nivel macro y muy pocos en escala nanométrica. En los estudios de las propiedades mecánicas, los nanotubos de carbono y grafeno se presen- tan como una alternativa, sin embargo, los estudios de biocompatibilidad son aún deficientes. Acerca de los estudios in vivo, sobre nanotubos de carbono, grafeno, óxido de titanio, nanopartículas de plata y nanopartículas de sílice mesoporosa existe poca información. Como anclaje para las nanopartículas se sugiere el material biocompatible hidroxiapatita, por el número de estudios que respaldan su uso. Como 
potencial biomaterial de recubrimiento se recomiendan a las nano- partículas de colágeno, por la compatibilidad a nivel tisular.

\section{AGRADECIMIENTOS}

Este estudio fue realizado como proyecto de la asignatura de Nanotecnologías y Materiales Funcionales para Diseño de la Maestría en Diseño Industrial y de Procesos de la Universidad Internacional SEK del
Ecuador en el año 2020. Los autores agradecen al Dr. Alberto Larrea, director del Proyecto Jubilous 3D de la Pontificia Universidad Católica del Ecuador.

\section{LISTA DE REFERENCIAS}

Ahn, E. Y., Jin, H., \& Park, Y. (2019). Assessing the antioxidant, cytotoxic, apoptotic and wound healing properties of silver nanoparticles green-synthesized by plant extracts. Materials Science and Engineering C, 101, 204-216. https://doi.org/10. 1016/j.msec.2019.03.095

Akbari, A., Majumder, M., \& Tehrani, A. (2015). Polylactic Acid (PLA) Carbon Nanotube Nanocomposites. In Handbook of Polymer Nanocomposites. Processing, Performance and Application (pp. 283-297). Springer Berlin Heidelberg. https://doi. org/10.1007/978-3-642-45229-1_45

Amarnath, C. A., Nanda, S. S., Papaefthymiou, G. C., Yi, D. K., \& Paik, U. (2013). Nanohybridization of Low-Dimensional Nanomaterials: Synthesis, Classification, and Application. Critical Reviews in Solid State and Materials Sciences, 38(1), 156. https://doi.org/10.1080/10408436.2012.732545

Anand Kumar, S., \& Shivraj Narayan, Y. (2019). Tensile Testing and Evaluation of 3DPrinted PLA Specimens as per ASTM D638 Type IV Standard. In U. et al. Chandrasekhar (Ed.), Innovative Design, Analysis and Development Practices in Aerospace and Automotive Engineering (I-DAD 2018) (pp. 79-95). Springer Nature Singapore Pte Ltd. https://doi.org/10.1007/978-981-13-2718-6_9 
Andrade Guel, M. L., López López, L. I., \& Sáenz Galindo, A. (2012). Nanotubos de carbono: funcionalización y aplicaciones biológicas. Revista Mexicana de Ciencias Farmacéuticas, 43(3).

Ángel-lópez, H. Z. Del. (2017). Biopolímero reforzado con nanotubos de carbono para su aplicación como andamios en la Regeneración de Tejido Óseo. In Revistacid.Itslerdo.Edu.Mx (Issue 6501). http://revistacid.itslerdo.edu.mx/coninci2017/ 32 Biopolímero reforzado con nanotubos de carbono para su.pdf

ArthritisFoundation. (2016). Cirugía: Tipos de reemplazos. All You Need to Know About Joint Surgery. http://espanol.arthritis.org/espanol/tratamientos/cirugia/cirugiatipos-reemplazos/

Asefa, T., \& Tao, Z. (2012). Biocompatibility of Mesoporous Silica Nanoparticles. Chemical Research in Toxicology, 25(11), 2265-2284. https://doi.org/10.1021/ tx300166u

Auras, R., Harte, B., \& Selke, S. (2004). An Overview of Polylactides as Packaging Materials. Macromolecular Bioscience, 4(9), 835-864. https://doi.org/10.1002/mabi .200400043

Botia Prada, G. C., \& Orjuela Abril, M. S. (2016). Desarrollo de Green Thermosets: Nueva Tecnología. Tercer Encuentro Internacional Universidad - Empresa En EI Sector de La Ingeniería.

Cakir, S., Aycicek, M., \& Akinci, A. (2018). Investigation of the mechanical and physical properties of PLA produced by injection molding for matrix material of polymer composites. Materials Science: Advanced Composite Materials, 2(1). https://doi. org/10.18063/msacm.v2i1.607

Carmona, E. R., Plaza, T., Recio-Sánchez, G., \& Parodi, J. (2018). Generation of a protocol for the synthesis of chitosan nanoparticles loaded with florfenicol through the ionic gelation method. Revista de Investigaciones Veterinarias Del Peru, 29(4), 1195-1202. https://doi.org/10.15381/rivep.v29i4.15203 
Casalini, T., Rossi, F., Castrovinci, A., \& Perale, G. (2019). A Perspective on Polylactic Acid-Based Polymers Use for Nanoparticles Synthesis and Applications. Frontiers in Bioengineering and Biotechnology, 7. https://doi.org/10.3389/fbioe.2019. 00259

Chen, X., \& Schluesener, H. J. (2008). Nanosilver: A nanoproduct in medical application. Toxicology Letters, 176(1), 1-12. https://doi.org/10.1016/j.toxlet.2007. 10.004

Chomoucka, J., Drbohlavova, J., Huska, D., Adam, V., Kizek, R., \& Hubalek, J. (2010). Magnetic nanoparticles and targeted drug delivering. Pharmacological Research, 62(2), 144-149. https://doi.org/10.1016/j.phrs.2010.01.014

Colorado, A. C., Agudelo, C. A., \& Moncada, M. E. (2013). Análisis de biomateriales para uso en ingeniería de tejido de piel: revisión. Revista Ingeniería Biomédica, 7(14), 11-23.

da Silva, D., Kaduri, M., Poley, M., Adir, O., Krinsky, N., Shainsky-Roitman, J., \& Schroeder, A. (2018). Biocompatibility, biodegradation and excretion of polylactic acid (PLA) in medical implants and theranostic systems. Chemical Engineering Journal, 340, 9-14. https://doi.org/10.1016/j.cej.2018.01.010

DeStefano, V., Khan, S., \& Tabada, A. (2020). Applications of PLA in modern medicine. Engineered Regeneration, 1(August), 76-87. https://doi.org/10.1016/j.engreg. 2020.08.002

Dev, A., Binulal, N. S., Anitha, A., Nair, S. V, Furuike, T., Tamura, H., \& Jayakumar, R. (2010). Preparation of poly(lactic acid)/chitosan nanoparticles for anti-HIV drug delivery applications. Carbohydrate Polymers, 80(3), 833-838. https://doi.org/ 10.1016/j.carbpol.2009.12.040

Dresselhaus, M. S., Dresselhaus, G., Eklund, P. C., \& Rao, A. M. (2000). Carbon Nanotubes. In W. Andreoni (Ed.), The Physics of Fullerene-Based and Fullerene-Related Materials (pp. 331-379). Springer Netherlands. https://doi.org/10.1007 1978-94-011-4038-6_9 
Dufresne, A. (2013). Nanocellulose: a new ageless bionanomaterial. Materials Today, 16(6), 220-227. https://doi.org/10.1016/j.mattod.2013.06.004

Ee, L. Y., \& Yau Li, S. F. (2021). Recent advances in 3D printing of nanocellulose: structure, preparation, and application prospects. Nanoscale Advances, 3(5), 11671208. https://doi.org/10.1039/D0NA00408A

El-Ghaouth, A., Smilanick, J. L., \& Wilson, C. L. (2000). Enhancement of the performance of Candida saitoana by the addition of glycolchitosan for the control of postharvest decay of apple and citrus fruit. Postharvest Biology and Technology, 19(1), 103-110. https://doi.org/10.1016/S0925-5214(00)00076-4

Enyashin, A. N., \& Ivanovskii, A. L. (2007). Functionalization of carbon nanotubes by covalently bonded graphite nanoplatelets: a theoretical study. Mendeleev Communications, 17(4), 199-201. https://doi.org/10.1016/j.mencom.2007.06.003

Facca, S., Lahiri, D., Fioretti, F., Messadeq, N., Mainard, D., Benkirane-Jessel, N., \& Agarwal, A. (2011). In vivo osseointegration of nano-designed composite coatings on titanium implants. ACS Nano, 5(6), 4790-4799. https://doi.org/10.1021/ nn200768c

Farah, S., Anderson, D. G., \& Langer, R. (2016). Physical and mechanical properties of PLA, and their functions in widespread applications - A comprehensive review. Advanced Drug Delivery Reviews, 107, 367-392. https://doi.org/10.1016/j. addr.2016.06.012

French, A. D. (2014). Idealized powder diffraction patterns for cellulose polymorphs. Cellulose, 21(2), 885-896. https://doi.org/10.1007/s10570-013-0030-4

Ghasemi, R., Abdollahi, M., Emamgholi Zadeh, E., Khodabakhshi, K., Badeli, A., Bagheri, H., \& Hosseinkhani, S. (2018). mPEG-PLA and PLA-PEG-PLA nanoparticles as new carriers for delivery of recombinant human Growth Hormone (rhGH). Scientific Reports, 8(1), 9854. https://doi.org/10.1038/s41598-018-28092-8

Gherasim, O., Grumezescu, A. M., Grumezescu, V., lordache, F., Vasile, B. S., \& Holban, A. M. (2020). Bioactive Surfaces of Polylactide and Silver Nanoparticles for the Prevention of Microbial Contamination. Materials, 13(3), 768. https://doi.org/ 10.3390/ma13030768 
Gómez Blázquez, G. (2019). Proyecto de Diseño de una Prótesis a Partir de Fabricación Aditiva (Impresión 3D).

Gong, D., Grimes, C. A., Varghese, O. K., Hu, W., Singh, R. S., Chen, Z., \& Dickey, E. C. (2001). Titanium oxide nanotube arrays prepared by anodic oxidation. Journal of Materials Research, 16(12), 3331-3334. https://doi.org/10.1557/JMR.2001. 0457

Göpferich, A. (1996). Mechanisms of polymer degradation and erosion. Biomaterials, 17(2), 103-114. https://doi.org/10.1016/0142-9612(96)85755-3

Gregor, A., Filová, E., Novák, M., Kronek, J., Chlup, H., Buzgo, M., Blahnová, V., Lukášová, V., Bartoš, M., Ne as, A., \& Hošek, J. (2017). Designing of PLA scaffolds for bone tissue replacement fabricated by ordinary commercial 3D printer. Journal of Biological Engineering, 11(1), 31. https://doi.org/10.1186/s13036-0170074-3

Gumrah Dumanli, A. (2017). Nanocellulose and its Composites for Biomedical Applications. Current Medicinal Chemistry, 24(5), 512-528. https://doi.org/10.2174/ 0929867323666161014124008

Hernández-Cocoletzi, H., Águila Almanza, E., Flores Agustin, O., Viveros Nava, E. L., \& Ramos Cassellis, E. (2009). Obtención y Caracterización de Quitosano a Partir de Exoesqueletos de Camarón. Superficies y Vacío, 22(3), 57-60.

Hou, A.-L., \& Qu, J.-P. (2019). Super-Toughened Poly(lactic Acid) with Poly(־-caprolactone) and Ethylene-Methyl Acrylate-Glycidyl Methacrylate by Reactive Melt Blending. Polymers, 11(5), 771. https://doi.org/10.3390/polym11050771

Huang, X., Qi, X., Boey, F., \& Zhang, H. (2012). Graphene-based composites. Chem. Soc. Rev., 41(2), 666-686. https://doi.org/10.1039/C1CS15078B

Jiang, T., James, R., Kumbar, S. G., \& Laurencin, C. T. (2014). Chitosan as a Biomaterial: Structure, Properties, and Applications in Tissue Engineering and Drug Delivery. In Natural and Synthetic Biomedical Polymers (pp. 91-113). Elsevier. https://doi.org/10.1016/B978-0-12-396983-5.00005-3 
Kalarikkal, N., Augustine, R., Oluwafemi, O. S., K. S., J., \& Thomas, S. (2016). Nanomedicine and Tissue Engineering: State of the Art and Recent Trends. Apple Academic Press, Inc.

Kasuga, T., Hiramatsu, M., Hoson, A., Sekino, T., \& Niihara, K. (1998). Formation of Titanium Oxide Nanotube. Langmuir, 14(12), 3160-3163. https://doi.org/10.1021/ la9713816

Khanna, P. K., Singh, N., Charan, S., Subbarao, V. V. V. S., Gokhale, R., \& Mulik, U. P. (2005). Synthesis and characterization of Ag/PVA nanocomposite by chemical reduction method. Materials Chemistry and Physics, 93(1), 117-121. https://doi. org/10.1016/j.matchemphys.2005.02.029

Komoto, D., Ikeda, R., Furuike, T., \& Tamura, H. (2018). Preparation of Chitosan-Coated Poly(L-Lactic Acid) Fibers for Suture Threads. Fibers, 6(4), 84. https://doi.org/ 10.3390/fib6040084

Kucharczyk, P., Pavelková, A., Stloukal, P., \& Sedlarík, V. (2016). Degradation behaviour of PLA-based polyesterurethanes under abiotic and biotic environments. Polymer Degradation and Stability, 129, 222-230. https://doi.org/10.1016/j.polymdegradstab.2016.04.019

Kumar, N., Chauhan, N. S., Mittal, A., \& Sharma, S. (2018). TiO2 and its composites as promising biomaterials: a review. BioMetals, 31(2), 147-159. https://doi.org/ 10.1007/s10534-018-0078-6

Liao, C., Li, Y., \& Tjong, S. C. (2019). Antibacterial Activities of Aliphatic Polyester Nanocomposites with Silver Nanoparticles and/or Graphene Oxide Sheets. Nanomaterials, 9(8), 1102. https://doi.org/10.3390/nano9081102

Lin, N., \& Dufresne, A. (2014). Nanocellulose in biomedicine: Current status and future prospect. European Polymer Journal, 59, 302-325. https://doi.org/10.1016/j.eurpolymj.2014.07.025

Liu, W., Zhou, J., Ma, Y., Wang, J., \& Xu, J. (2017). Fabrication of PLA Filaments and its Printable Performance. IOP Conference Series: Materials Science and Engineering, 275, 12033. https://doi.org/10.1088/1757-899X/275/1/012033 
Lu, H. W., Liu, S. H., Wang, X. L., Qian, X. F., Yin, J., \& Zhu, Z. K. (2003). Silver nanocrystals by hyperbranched polyurethane-assisted photochemical reduction of Ag+. Materials Chemistry and Physics, 81(1), 104-107. https://doi.org/10.1016/ S0254-0584(03)00147-0

Mediforum. (2016). La nanotecnología es el nuevo gran aliado odontológico.

Moniruzzaman, M., \& Winey, K. I. (2006). Polymer Nanocomposites Containing Carbon Nanotubes. Macromolecules, 39(16), 5194-5205. https://doi.org/10.1021/ ma060733p

Nagarajan, V., Mohanty, A. K., \& Misra, M. (2016). Perspective on Polylactic Acid (PLA) based Sustainable Materials for Durable Applications: Focus on Toughness and Heat Resistance. ACS Sustainable Chemistry \& Engineering, 4(6), 2899-2916. https://doi.org/10.1021/acssuschemeng.6b00321

Ñauta Ñauta, A. E., \& Vergara Idrovo, M. R. (2017). Simulación de Termofluidos de PLA Natural y ABS en el Proceso de Impresión en 3D.

Oksiuta, Z., Jalbrzykowski, M., Mystkowska, J., Romanczuk, E., \& Osiecki, T. (2020). Mechanical and Thermal Properties of Polylactide (PLA) Composites Modified with Mg, Fe, and Polyethylene (PE) Additives. Polymers, 12(12), 2939. https://doi. org/10.3390/polym12122939

Parwe, S. P., Chaudhari, P. N., Mohite, K. K., Selukar, B. S., Nande, S. S., \& Garnaik, B. (2014). Synthesis of ciprofloxacin-conjugated poly (L-lactic acid) polymer for nanofiber fabrication and antibacterial evaluation. International Journal of $\mathrm{Na}$ nomedicine, 1463. https://doi.org/10.2147/JJN.S54971

Peters, R. J. B., van Bemmel, G., Herrera-Rivera, Z., Helsper, H. P. F. G., Marvin, H. J. P., Weigel, S., Tromp, P. C., Oomen, A. G., Rietveld, A. G., \& Bouwmeester, H. (2014). Characterization of Titanium Dioxide Nanoparticles in Food Products: Analytical Methods To Define Nanoparticles. Journal of Agricultural and Food Chemistry, 62(27), 6285-6293. https://doi.org/10.1021/j55011885

Petersen, D. K., Naylor, T. M., \& Halen, J. P. Ver. (2014). Current and future applications of nanotechnology in plastic and reconstructive surgery. Plastic and Aesthetic Research, 1, 43-50. https://doi.org/10.4103/2347-9264.139698 
Pinto, A. M., Moreira, S., Gonçalves, I. C., Gama, F. M., Mendes, A. M., \& Magalhães, F. D. (2013). Biocompatibility of poly(lactic acid) with incorporated graphenebased materials. Colloids and Surfaces B: Biointerfaces, 104, 229-238. https://doi.org/10.1016/j.colsurfb.2012.12.006

Qiu, T. Y., Song, M., \& Zhao, L. G. (2016). Testing, characterization and modelling of mechanical behaviour of poly (lactic-acid) and poly (butylene succinate) blends. Mechanics of Advanced Materials and Modern Processes, 2(1), 7. https://doi. org/10.1186/s40759-016-0014-9

Rajapakse, R. M. G., Wijesinghe, W. P. S. L., Mantilaka, M. M. M. G. P. G., Chathuranga Senarathna, K. G., Herath, H. M. T. U., Premachandra, T. N., Ranasinghe, C. S. K., Rajapakse, R. P. V. J., Edirisinghe, M., Mahalingam, S., Bandara, I. M. C. C. D., \& Singh, S. (2016). Preparation of bone-implants by coating hydroxyapatite nanoparticles on self-formed titanium dioxide thin-layers on titanium metal surfaces. Materials Science and Engineering C, 63, 172-184. https://doi.org/10. 1016/j.msec.2016.02.053

Roberts, G. A. F. (1992). Structure of Chitin and Chitosan. In Chitin Chemistry (pp. 153). Macmillan Education UK. https://doi.org/10.1007/978-1-349-11545-7_1

Sangeetha, V., Sudha, P. N., Gomathi, T., \& Jayaprabakar, J. (2019). Fabrication of nanochitosan based biocompatible polymer blend for bone tissue engineering applications. Digest Journal of Nanomaterials and Biostructures, 14(4), 963-972.

Segura González, E. A. (2016). Diseño, caracterización y comportamiento en servicio de materiales basados en ácido poliláctico ( PLA ) con potencial utilidad en el empaquetado de alimentos. http://hdl.handle.net/10016/23514

Sha, L., Chen, Z., Chen, Z., Zhang, A., \& Yang, Z. (2016). Polylactic Acid Based Nanocomposites: Promising Safe and Biodegradable Materials in Biomedical Field. International Journal of Polymer Science, 2016, 1-11. https://doi.org/10. $1155 / 2016 / 6869154$

Shebi, A., \& Lisa, S. (2019). Evaluation of biocompatibility and bactericidal activity of hierarchically porous PLA-TiO2 nanocomposite films fabricated by breath-figure method. Materials Chemistry and Physics, 230, 308-318. https://doi.org/ 10.1016/j.matchemphys.2019.03.045 
Sonseca, A., Madani, S., Rodríguez, G., Hevilla, V., Echeverría, C., Fernández-García, M., Muñoz-Bonilla, A., Charef, N., \& López, D. (2019). Multifunctional PLA Blends Containing Chitosan Mediated Silver Nanoparticles: Thermal, Mechanical, Antibacterial, and Degradation Properties. Nanomaterials (Basel, Switzerland), 10(1). https://doi.org/10.3390/nano10010022

Tanpichai, S., \& Wootthikanokkhan, J. (2016). Reinforcing abilities of microfibers and nanofibrillated cellulose in poly(lactic acid) composites. Science and Engineering of Composite Materials, 25. https://doi.org/10.1515/secm-2016-0113

Vargas Pérez, J. L., Yunga Patiño, L. F., Cajamarca Guambaña, L. F., \& Matute Salinas, J. L. (2015). Diseño, construcción e implementación de prótesis biomecánica de mano derecha.

Velasco Nieto, D. (2016). Desarrollo de biomateriales celulares en base EVA, PLA Y PHB: fabricación y caracterización. https://doi.org/10.35376/10324/22235

Vyazovkin, S. (2020). Kissinger Method in Kinetics of Materials: Things to Beware and Be Aware of. Molecules, 25(12), 2813. https://doi.org/10.3390/molecules 25122813

Wassel, R. A., Grady, B., Kopke, R. D., \& Dormer, K. J. (2007). Dispersion of super paramagnetic iron oxide nanoparticles in poly(d,I-lactide-co-glycolide) microparticles. Colloids and Surfaces A: Physicochemical and Engineering Aspects, 292(2-3), 125-130. https://doi.org/10.1016/j.colsurfa.2006.06.012

Xiang, H., Mu, Y., Hu, C., \& Luo, X. (2017). Biocompatibility and Toxicity of Polylactic Acid/Ferrosoferric Oxide Nanomagnetic Microsphere. Journal of Nanomaterials, 2017, 1-8. https://doi.org/10.1155/2017/5429063

Xu, C., Lei, C., \& Yu, C. (2019). Mesoporous Silica Nanoparticles for Protein Protection and Delivery. Frontiers in Chemistry, 7, 290. https://doi.org/10.3389/fchem. 2019.00290

Xu, L., Crawford, K., \& Gorman, C. B. (2011). Effects of Temperature and pH on the Degradation of Poly(lactic acid) Brushes. Macromolecules, 44(12), 4777-4782. https://doi.org/10.1021/ma2000948 
Yan, Q., Lei, Y., \& Yuan, J. (2010). Preparation of titanium dioxide compound pigments based on kaolin substrates. Journal of Coatings Technology and Research, 7(2), 229-237. https://doi.org/10.1007/s11998-009-9172-6

Zaitsev, V. S., Filimonov, D. S., Presnyakov, I. A., Gambino, R. J., \& Chu, B. (1999). Physical and Chemical Properties of Magnetite and Magnetite-Polymer Nanoparticles and Their Colloidal Dispersions. Journal of Colloid and Interface Science, 212(1), 49-57. https://doi.org/10.1006/jcis.1998.5993

Zhang, Z. (2001). A convenient route to polyacrylonitrile/silver nanoparticle composite by simultaneous polymerization-reduction approach. Polymer, 42(19), 83158318. https://doi.org/10.1016/S0032-3861(01)00285-3

Zheng, M., Gu, M., Jin, Y., \& Jin, G. (2001). Optical properties of silver-dispersed PVP thin film. Materials Research Bulletin, 36(5-6), 853-859. https://doi.org/10. 1016/S0025-5408(01)00525-6

Zheng, X., Zhou, S., Xiao, Y., Yu, X., Li, X., \& Wu, P. (2009). Shape memory effect of poly(d,I-lactide)/Fe3O4 nanocomposites by inductive heating of magnetite particles. Colloids and Surfaces B: Biointerfaces, 71(1), 67-72. https://doi.org/10. 1016/j.colsurfb.2009.01.009

Zhou, C., Shi, Q., Guo, W., Terrell, L., Qureshi, A. T., Hayes, D. J., \& Wu, Q. (2013). Electrospun Bio-Nanocomposite Scaffolds for Bone Tissue Engineering by Cellulose Nanocrystals Reinforcing Maleic Anhydride Grafted PLA. ACS Applied Materials \& Interfaces, 5(9), 3847-3854. https://doi.org/10.1021/am4005072

Ziabką, M., Menaszek, E., Tarasiuk, J., \& Wroński, S. (2018). Biocompatible nanocomposite implant with silver nanoparticles for otology_-In vivo evaluation. Nanomaterials, 8(10). https://doi.org/10.3390/nano8100764 